Paper:

\title{
Parallel Distributed Compensation Based Stabilization of A 3-DOF RC Helicopter: A Tensor Product Transformation Based Approach
}

\author{
Péter Baranyi* ${ }^{*}$ Péter Korondi**, and Kazuo Tanaka*** \\ *Computer and Automation Research Institute of the Hungarian Academy of Sciences \\ H-1111 Budapest, Kende u. 13-17, Hungary \\ E-mail: baranyi@sztaki.hu \\ ${ }^{* *}$ Department of Automation and Applied Informatics, Budapest University of Technology and Economics, Hungary \\ E-mail: korondi@elektro.get.bme.hu \\ *** Department of Mechanical Engineering and Intelligent Systems, The University of Electro-Communications \\ Tokyo 182-8585, Japan \\ E-mail:ktanaka@mce.uec.ac.jp \\ [Received January 10, 2008; accepted April 25, 2008]
}

\begin{abstract}
This paper presents a control solution for the stabilization of the 3-DOF RC helicopter via the combination of the TP (Tensor Product) model transformation and the PDC (Parallel Distributed Compensation) control design framework. First we recall the nonlinear model of the RC helicopter and its simplified version, in order, to facilitate control design. Then we execute the TP model transformation on the simplified model to yield its TP model representation, that is a kind of polytopic model with specific characteristics, whereupon the PDC framework can immediately be applied. The control design considers practical control specifications such as: good speed of response and physical constrain on the control effort to avoid actuator saturations. We guarantee these specifications by LMI (Linear Matrix Inequality) conditions developed under the PDC frameworks. Further, we avoid the discrepancies, introduced via the simplification of the model, by applying LMI conditions specialized for robust control. By simultaneously solving these LMI conditions, we render a stabilizing nonlinear controller that achieves good speed of response with small control effort without actuator saturations. Simulation results are included to validate the control design. It will be pointed out that the resulting controller is equivalent with the controller successfully applied in the real control experiment of the helicopter presented in a recent paper.

The main conclusion of this paper is, that the proposed design process is systematic, non-heuristic and straightforward, the stability proof of the resulting controller is tractable via the feasibility test of LMIs and, hence, exact. The whole design procedure is automatically computed via commercialized mathematical tools (MATLAB LMI Toolbox) without analytical interaction. The computational time is about minutes on a regular PC.
\end{abstract}

Keywords: 3-DOF helicopter, robust control, linear parameter-varying (LPV) model, TP model transformation, parallel distributed compensation (PDC), linear matrix inequalities (LMI).

\section{Introduction}

Modern control theories mainly focuses on analysis and control design based on LMIs in LPV system representation. These control methodologies offer a well developed, rather tractable, mathematically non-heuristic ways to derive controllers. In the same time various new identification and control design tools are emerging utilizing representation forms far from the classical analytic formulas (e.g. neural network, fuzzy logic, genetic algorithms etc.). The number of papers using these techniques even in industrial applications are rapidly increasing [1-7]. However, the key problem is here to find a uniform gateway between the well developed LPV and LMI based design methods based on analytical formulas and the modern identification techniques resulting in different type of representations. As a solution this paper proposes a control methodology that is a combination of two numerical methods such as the TP model transformation [8] and the PDC control design framework [9].

The TP model transformation is developed to uniformly transform a given Linear Parameter Varying (LPV) model (from a large class of LPV models) to TP model polytopic form, namely, to a parameter varying convex combination of LTI linear time invariant (vertex) systems. It is irrelevant whether the given LPV model represents a physical model, or is just the outcome of black-box identification (e.g. neural net model, various fuzzy models). The TP model transformation replaces the analytical affine and polytopic decomposition techniques by a uniform problem independent numerical method, and offers options to generate various types of convex combina- 
tions which can hardly be determined or even cannot be achieved via analytical derivations in general [10]. These different convex combinations lead to different representation of the given model, and, hence, to different advantages of the further LMI design. The resulting TP model satisfies all the characteristics required by the PDC design framework, so as it can immediately be executed on the resulting TP model. Recent papers further discusse the philosophy of TP model based control from different aspects [17-25].

The PDC framework [9] starts with a given TP model and results in a nonlinear controller. The key step of the PDC design is to substitute the LTI systems of the TP model into LMI conditions selected according to various, multi-objective, control design specifications. The solution of the LMI conditions directly leads to the controller. The PDC framework is a numerical design procedure and can be automatically executed on regular computers.

In conclusion the proposed combination of the these two concepts can be automatically executed without analytical interaction one after other in a few minutes (on a regular PC). If the LPV model is modified then the design process simply can be repeated on the same way. Further, this design provides a straightforward systematic procedure for nonlinear control design with guaranteed stability and multi-objective performance. The stability proof of the resulting controller is exact and tractable via the LMI conditions. The prosed design can be rather easily implemented in mathematical programming tools (we refer to TP Tool, tptool.sztaki.hu, where a complete MATLAB Toolbox is given for this concept). The key idea of this design methodology was proposed in [8]. We also refer the readers to other papers and special conference sessions which present solutions to realistic challenging control problems via TP model transformation and PDC [10]. Papers [11, 12] present observer and controller designs to stabilize the prototypical aeroelastic wing section with various control specifications.

The main objective of this paper is to investigate the effectiveness of the combination of the TP model transformation and PDC design framework through the task of controlling a real 3-DOF RC helicopter. We consider the following control specifications via properly selected LMI conditions: a) we define a decay rate control (to achieve good speed of response), b) we have practical constrain on the control value since each propeller rotates in one direction only (this explicitly address the avoidance of actuator saturations), c) we design robust controller to avoid the discrepancies caused by the model simplification. This paper shows that the resulting controller satisfies the desired control specifications. We present simulation results only, but we point on the fact that the resulting controller is equivalent to the one derived in [13] and successfully tested in laboratory experiment.

The paper is organized as follows: Section 3 presents the uncertain LPV model of the 3-DOF RC helicopter. Section 4 discuses the TP model transformation and the PDC design with special focus on the present model of the helicopter. Section 5 derives the TP model of the he-

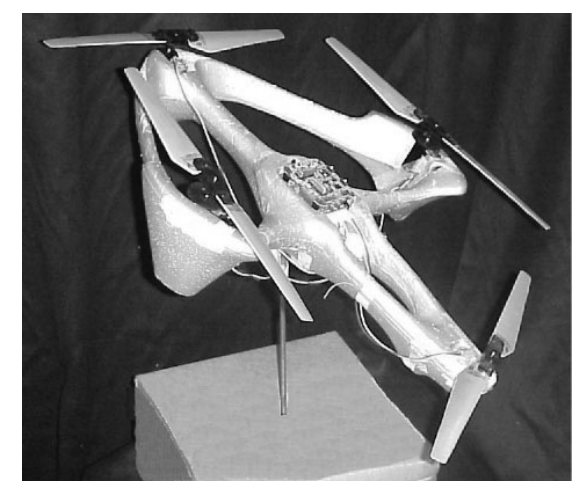

Fig. 1. RC helicopter.

licopter and shows that the equations of motion can be exactly represented as a convex combination of four LTI systems. This section also derives the controller via solving LMI conditions selected according to the control specifications. The detailed internal numerical results are also presented. Section 6 presents the control results. Finally section 6 concludes the paper.

\section{Nomenclature}

- $\alpha=$ yaw angle

- $\beta=$ pitch angle

- $\gamma=$ roll angle

- $I_{\gamma, \beta, \alpha}=$ the moments of inertia around $x, y$ and $z$ axes with respect to the gravity point of the helicopter.

- $M=$ mass of the helicopter

- $g=$ is the gravity constant

- $e, l_{1}, l_{2}=$ lengths of the body of the helicopter as shown on the figures

- $F_{i}=$ lift force generated by the $i$-th propeller

- $C_{L}=$ lift coefficient

- $\rho=$ air density

- $l_{w}=$ length of a wing of a propeller

- $\theta_{i}=$ angle of the $i$-th propeller

- $C_{r}, C_{p}, C_{u r}, C_{u p}, C_{u y}=$ constants consisting $M, I_{\gamma}, I_{\beta}, I_{\alpha}, g, e, l_{1}, l_{2}, C_{L}, \rho, S$

\section{Equations of Motion of the RC Helicopter Dynamics}

Figure 1 is a picture of a 3-DOF RC helicopter. One can see that the degrees of freedom are reduced by fixing at a (joint) point. Figs. 2, 3, 4, 5 and 6 present the schematic drawings and the physical parameters, the pitch, roll and yaw modes of the RC helicopter. The 


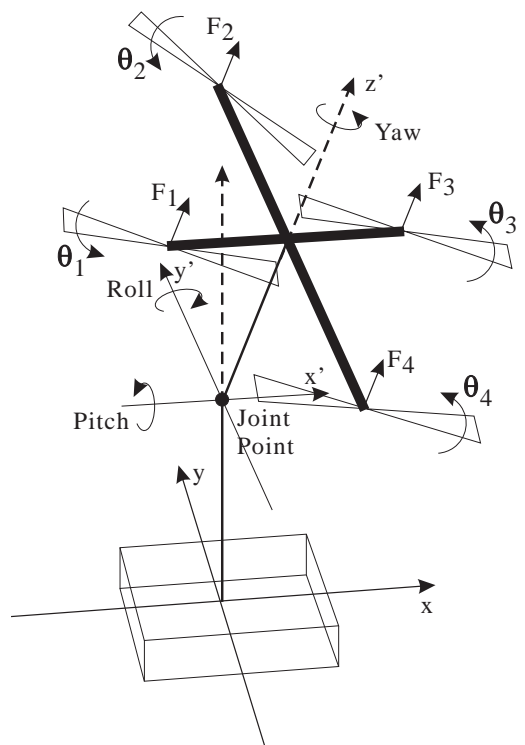

Fig. 2. Helicopter model fixed at a joint point.

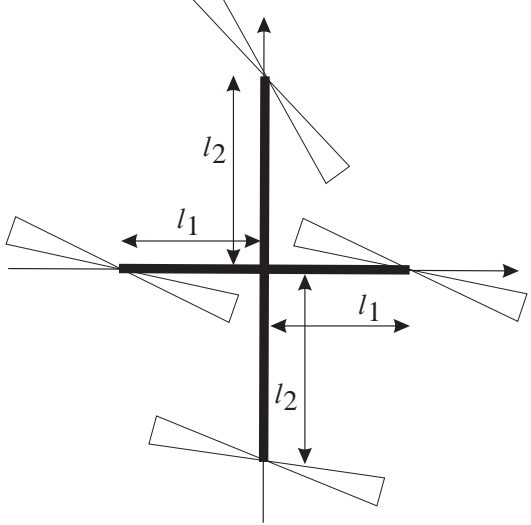

Fig. 3. Helicopter with four propellers.

derivation of the equations of motion is given in the Appendix $B$ and also detailed in [13]. The result of the derivation is:

$$
\begin{aligned}
\ddot{\gamma}(t)= & C_{r} \sin \gamma(t)+C_{u r}\left(2 \Omega+\Delta \dot{\theta}_{1}(t)+\Delta \dot{\theta}_{3}(t)\right) \\
& \times\left(\Delta \dot{\theta}_{1}(t)-\Delta \dot{\theta}_{3}(t)\right) \quad . \quad . . . . \\
\ddot{\beta}(t)= & C_{p} \sin \beta(t)+C_{u p}\left(2 \Omega+\Delta \dot{\theta}_{2}(t)+\Delta \dot{\theta}_{4}(t)\right) \\
& \times\left(-\Delta \dot{\theta}_{2}(t)+\Delta \dot{\theta}_{4}(t)\right) . . . . . . \\
\dot{\alpha}(t)= & C_{u y}\left(\Delta \dot{\theta}_{1}(t)+\Delta \dot{\theta}_{3}(t)-\Delta \dot{\theta}_{2}(t)-\Delta \dot{\theta}_{4}(t)\right) .
\end{aligned}
$$

\subsection{Simplified Model}

To facilitate the controller design we attempt to simplify the nonlinear model. By considering the assumption that $\Delta \dot{\theta}_{1}(t)+\Delta \dot{\theta}_{3}(t)$ and $\Delta \dot{\theta}_{2}(t)+\Delta \dot{\theta}_{4}(t)$ are small, (12) and (13) can be approximated as

$$
\begin{aligned}
\ddot{\gamma}(t) & =C_{r} \sin \gamma(t)+2 C_{u r} \Omega\left(\Delta \dot{\theta}_{1}(t)-\Delta \dot{\theta}_{3}(t)\right) . \\
\ddot{\beta}(t) & =C_{p} \sin \beta(t)+2 C_{u p} \Omega\left(-\Delta \dot{\theta}_{2}(t)+\Delta \dot{\theta}_{4}(t)\right) .
\end{aligned}
$$

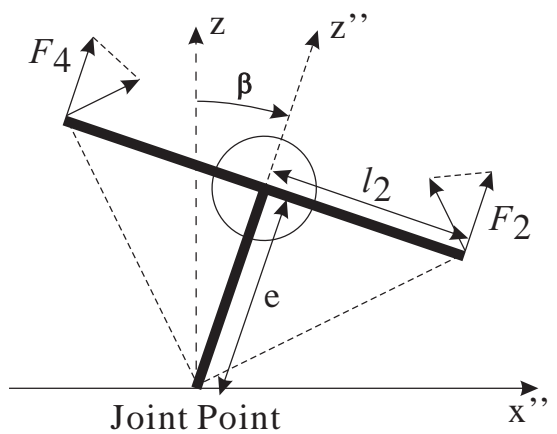

Fig. 4. Pitch.

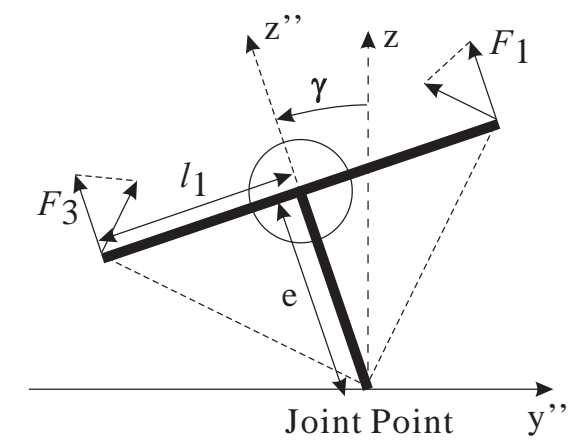

Fig. 5. Roll.

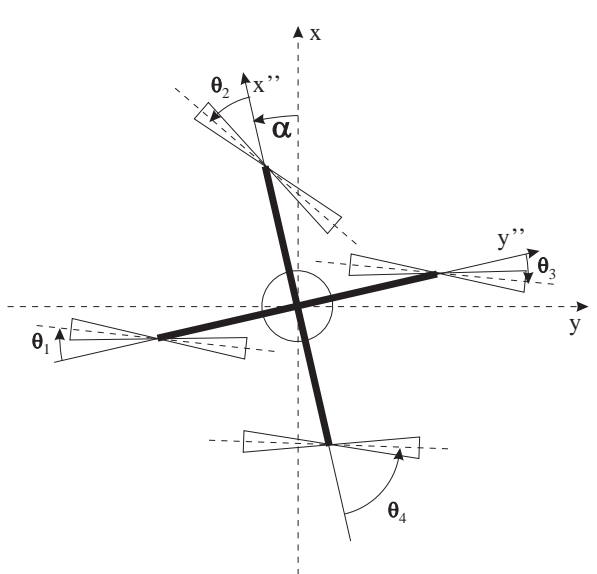

Fig. 6. Yaw.

If we define state-vector

$$
\vec{x}(t)=\left(\begin{array}{c}
x_{1}(t) \\
x_{2}(t) \\
x_{3}(t) \\
x_{4}(t) \\
x_{5}(t)
\end{array}\right)=\left(\begin{array}{c}
\gamma(t) \\
\dot{\gamma}(t) \\
\beta(t) \\
\dot{\beta}(t) \\
\alpha(t)
\end{array}\right),
$$

and input vector

$$
\begin{aligned}
\vec{u}(t)=\left(\begin{array}{l}
u_{1}(t) \\
u_{2}(t) \\
u_{3}(t) \\
u_{4}(t)
\end{array}\right) & =\left(\begin{array}{c}
\Delta \dot{\theta}_{1}(t)-\Delta \dot{\theta}_{3}(t) \\
\Delta \dot{\theta}_{1}(t)+\Delta \dot{\theta}_{3}(t) \\
-\Delta \dot{\theta}_{2}(t)+\Delta \dot{\theta}_{4}(t) \\
\Delta \dot{\theta}_{2}(t)+\Delta \dot{\theta}_{4}(t)
\end{array}\right) \\
& \simeq\left(\begin{array}{c}
\Delta \dot{\theta}_{1}(t)-\Delta \dot{\theta}_{3}(t) \\
0 \\
-\Delta \dot{\theta}_{2}(t)+\Delta \dot{\theta}_{4}(t) \\
0
\end{array}\right) .
\end{aligned}
$$


then we arrive at the following LPV model:

$$
\dot{\mathbf{x}}(t)=\mathbf{A}(\mathbf{p}(t)) \mathbf{x}(t)+\mathbf{B} \mathbf{u}(t) \ldots \ldots
$$

where $\mathbf{p}(t)=\left(\begin{array}{ll}x_{1}(t) & x_{3}(t)\end{array}\right)=\left(\begin{array}{ll}\gamma(t) & \beta(t)\end{array}\right)$.

\subsection{Involving Uncertainty}

The simplification (6) of the model causes some modeling discrepancies, therefore the controller designed for the simplified dynamics may not always stabilize the original nonlinear dynamics. Thus, we give an uncertainty model to compensate the modelling discrepancies.

From the above simplification (6) the modelling error is

$$
\left[\begin{array}{cccc}
0 & 0 & 0 & 0 \\
C_{u r} u_{2}(t) & 0 & 0 & 0 \\
0 & 0 & 0 & 0 \\
0 & 0 & C_{u p} u_{4}(t) & 0 \\
0 & 0 & 0 & 0
\end{array}\right] \mathbf{u}(t) \ldots .
$$

The modeling error (8) can be covered by the uncertain blocks of (14). The upper bounds $\frac{1}{\rho_{a}}$ and $\frac{1}{\rho_{b}}$ of these uncertain blocks are known in this case, i.e., $\left\|\Delta_{a}(t)\right\| \leq$ $\frac{1}{\rho_{a}}=0$ and $\left\|\Delta_{b}(t)\right\| \leq \frac{1}{\rho_{b}}=\sqrt{u_{2 \max }^{2}+u_{4 \max }^{2}} \cdot u_{2 \max }$ and $u_{4 \max }$ denote the maximum values of $\left|u_{2}(t)\right|$ and $\left|u_{4}(t)\right|$, respectively. These values correspond to the saturations of actuators (i.e., angular velocity of the propellers).

In conclusion:

$$
\begin{aligned}
& \forall t: \Delta_{a}(t)=\mathbf{D}_{a}=\mathbf{E}_{a}=0 \ldots \ldots \\
& \Delta_{b}(t)=\left[\begin{array}{cccc}
u_{2}(t) & 0 & 0 & 0 \\
0 & 0 & 0 & 0 \\
0 & 0 & u_{4}(t) & 0 \\
0 & 0 & 0 & 0
\end{array}\right], \\
& \mathbf{D}_{b}=\left[\begin{array}{cccc}
0 & 0 & 0 & 0 \\
C_{u r} & 0 & 0 & 0 \\
0 & 0 & 0 & 0 \\
0 & 0 & C_{u p} & 0 \\
0 & 0 & 0 & 0
\end{array}\right], \quad \mathbf{E}_{b}=\mathbf{I}_{4} .
\end{aligned}
$$

\section{Control Design Methodology}

\subsection{Uncertain Convex State-Space TP Model}

Consider LPV state-space model:

$$
\left(\begin{array}{l}
\dot{\mathbf{x}}(t) \\
\mathbf{y}(t)
\end{array}\right)=\mathbf{S}(\mathbf{p}(t))\left(\begin{array}{l}
\mathbf{x}(t) \\
\mathbf{u}(t)
\end{array}\right)
$$

with input $\mathbf{u}(t)$, output $\mathbf{y}(t)$, state vector $\mathbf{x}(t)$ and system matrix $\mathbf{S}(\mathbf{p}(t))$ that is a parameter-varying object, where $\mathbf{p}(t) \in \Omega$ is time varying $N$-dimensional parameter vector. The parameter vector $\mathbf{p}(t)$ is an element of the closed hypercube $\Omega=\left[a_{1}, b_{1}\right] \times\left[a_{2}, b_{2}\right] \times \cdots \times\left[a_{N}, b_{N}\right] \subset \mathbb{R}^{N}$, and can also include some elements of $\mathbf{x}(t)$ (we restrict our discussion to cases when $\mathbf{p}(t)$ does not contains any elements of $\mathbf{u}(t)$, see the dynamic model the helicopter). Let the size of the system matrix $\mathbf{S}(\mathbf{p}(t))$ is $O \times I$ in the followings.
The convex state-space TP model (TP model for brevity) describes the LPV state-space model for any parameter $\mathbf{p}(t)$ as the convex combination of LTI system matrices (a detailed discussion of tensor notations and operations used in the followings is given in [14]):

$$
\left(\begin{array}{l}
\dot{\mathbf{x}}(t) \\
\mathbf{y}(t)
\end{array}\right)=\mathscr{S} \stackrel{N}{\otimes} \mathbf{w}_{n}\left(p_{n}(t)\right)\left(\begin{array}{l}
\mathbf{x}(t) \\
\mathbf{u}(t)
\end{array}\right) .
$$

where row vector $\mathbf{w}_{n}\left(p_{n}\right) \in \mathbb{R}^{I_{n}} n=1, \ldots, N$ contains coefficient functions $w_{n, i_{n}}\left(p_{n}\right)$. Function $w_{n, j}\left(p_{n}(t)\right) \in[0,1]$ is the $j$-th univariate coefficient function defined on the $n$ th dimension of $\Omega$, and $p_{n}(t)$ is the $n$-th element of vector $\mathbf{p}(t) . I_{n}(n=1, \ldots, N)$ is the number of univariate coefficient functions used in the $n$-th dimension of the parameter vector $\mathbf{p}(t)$. The $(N+2)$-dimensional coefficient tensor $\mathscr{S} \in \mathbb{R}^{I_{1} \times I_{2} \times \cdots \times I_{N} \times O \times I}$ is constructed from LTI vertex systems $\mathbf{S}_{i_{1} i_{2} \ldots i_{N}} \in \mathbb{R}^{O \times I}$. The convex combination of the LTI vertex systems is ensured by the conditions:

$$
\begin{gathered}
\forall n \in[1, N], i, p_{n}(t): w_{n, i}\left(p_{n}(t)\right) \in[0,1] ; \ldots \\
\forall n \in[1, N], p_{n}(t): \sum_{i=1}^{I_{n}} w_{n, i}\left(p_{n}(t)\right)=1
\end{gathered}
$$

Remark 1: There are cases when the TP model (11), with finite number of LTI components, cannot represent the given LPV model. In such a case the TP model is used as an approximation only. In this regards see papers [15], where the approximation trade-off property of the TP model and the TP model transformation is investigated. In the present paper we will show that the TP model can exactly represent the LPV model of the helicopter.

For further details we refer to $[8,11]$.

The model of the helicopter has uncertainty that is involved in the LPV model as:

$$
\begin{aligned}
\dot{\mathbf{x}}(t)= & \left.\left(\mathbf{A}(\mathbf{p}(t))+\mathbf{D}_{a}(\mathbf{p}(t)) \Delta_{a}(t) \mathbf{E}_{a}(\mathbf{p}(t))\right)\right) \mathbf{x}(t) \\
& \left.+\left(\mathbf{B}(\mathbf{p}(t))+\mathbf{D}_{b}(\mathbf{p}(t)) \Delta_{b}(t) \mathbf{E}_{b}(\mathbf{p}(t))\right)\right) \mathbf{u}(t) .
\end{aligned}
$$

$\Delta_{a}(t)$ and $\Delta_{b}(t)$ are unknown uncertain blocks. $\mathbf{D}_{a}, \mathbf{E}_{a}$, $\mathbf{D}_{b}$ and $\mathbf{E}_{b}$ are known matrices to provide the uncertain elements.

In order to involve uncertainty, we extend the TP model (11) with the following terms:

$$
\begin{aligned}
& \mathbf{D}_{a / b}(\mathbf{p}(t))=\mathscr{D}_{a / b} \stackrel{N}{\otimes}_{n=1}^{N} \mathbf{w}_{n}\left(p_{n}(t)\right) \mathbf{x}(t) \ldots
\end{aligned}
$$

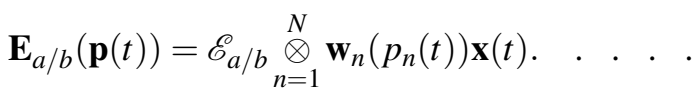

\subsection{TP Model Transformation}

The goal of the TP model transformation is to transform a given LPV state-space model into TP model (11) and $(15,16)$ with conditions $(12,13)$. The TP model transformation is capable of generating various different weighting functions to satisfy further conditions, see [10]. In order to have direct link between the TP model form and the typical LMI formulations derived under the PDC framework we simply transform the TP model into a typical 
polytopic form:

$$
\left(\begin{array}{l}
\dot{\mathbf{x}}(t) \\
\mathbf{y}(t)
\end{array}\right)=\sum_{r=1}^{R} w_{r}(\mathbf{p}(t)) \mathbf{S}_{r}\left(\begin{array}{l}
\mathbf{x}(t) \\
\mathbf{u}(t)
\end{array}\right) \ldots \ldots
$$

where

$$
\mathbf{S}_{r}=\left(\begin{array}{ll}
\mathbf{A}_{r} & \mathbf{B}_{r} \\
\mathbf{C}_{r} & \mathbf{D}_{r}
\end{array}\right)=\mathbf{S}_{i_{1}, i_{2}, \ldots, i_{N}},
$$

and $\mathbf{S}_{i_{1}, i_{2}, \ldots, i_{N}}$ are the elements of tensor $\mathscr{S}$ and $r=$ ordering $\left(i_{1}, i_{2}, \ldots, i_{N}\right)\left(r=1 . . R=\prod_{n} I_{n}\right)$. The function "ordering" results in the linear index equivalent of an $N$ dimensional array's index $i_{1}, i_{2}, \ldots, i_{N}$, when the size of the array is $I_{1} \times I_{2} \times \cdots \times I_{N}$. The coefficient functions are defined according to the sequence of $r$ as:

$$
w_{r}(\mathbf{p}(t))=\prod_{n} w_{n, i_{n}}\left(p_{n}(t)\right) .
$$

\subsection{PDC Framework}

The PDC design framework determines one LTI feedback gain to each LTI consequent system of the TP model or model (17). The PDC framework starts with the LTI vertex systems $\mathbf{S}_{r}$, and results in the vertex LTI feedback gains $\mathbf{F}_{r}$ of the controller. $\mathbf{F}_{r}$ is computed via solving the LMI conditions defined under the PDC framework. Having the $\mathbf{F}_{r}$ the control value is determined by the same weighting functions as used in (17):

$$
\mathbf{u}(t)=-\left(\sum_{r=1}^{R} w_{r}(\mathbf{p}(t)) \mathbf{F}_{r}\right) \mathbf{x}(t) \ldots \ldots
$$

The LMI conditions, to be solved under PDC framework, are selected according to the stability criteria and the desired control performance. In such a way, the speed of response, constraints on the state vector or on the control value can be considered via properly selected LMI based stability conditions [9].

\section{Control Design of the 3-DOF RC Helicopter}

First of all, let us define the design specifications:

- We derive a robust control since the model does not consider all real effects (for instance, the ground effect and propeller deflection are not considered in the model).

- We derive a decay rate control to have good speed of response.

- We have constrains on the control value (the physical operation range of the engine of propellers).

- We assume that the initial of the state vector is unknown.

- The design must consider the uncertain components of the LPV model.

In the followings we derive the polytopic model (17) by the TP model transformation, and in the second step we apply the PDC framework.
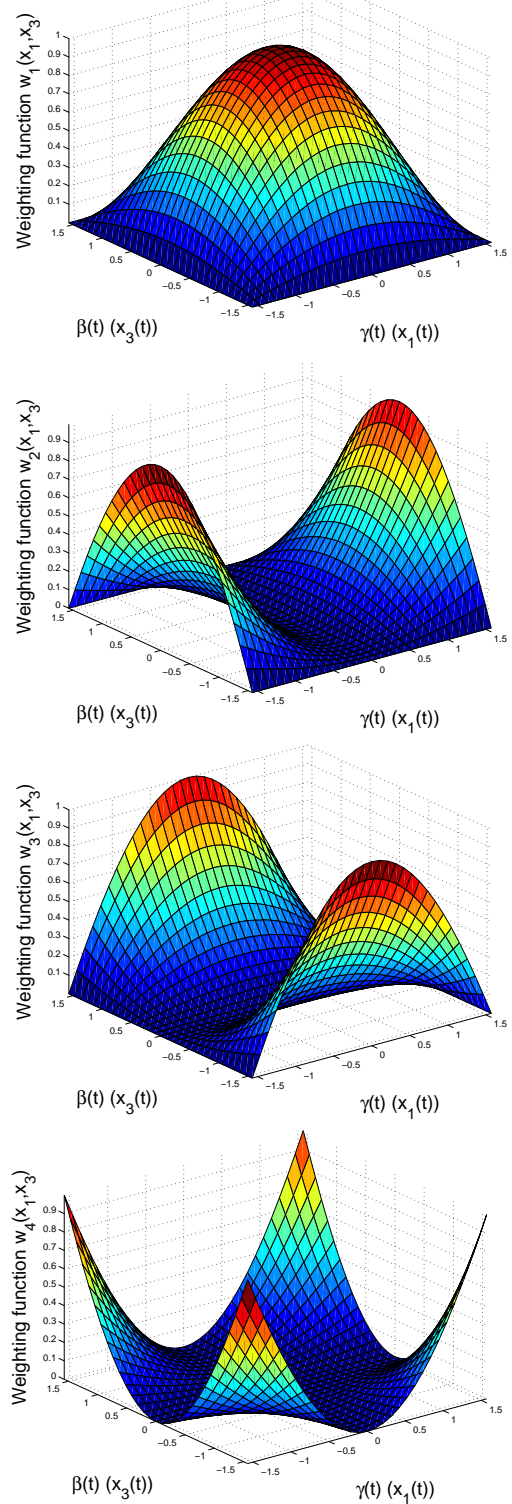

Fig. 7. Weighting functions $w_{r}\left(x_{1}, x_{2}\right)$ of roll angle $x_{1}(t)$ and pitch angle $x_{3}(t)$.

\subsection{Convex State-Space TP Model of the 3-DOF RC Helicopter by TP Model Transformation}

Let the transformation space $\Omega$ be set as $x_{1}(t) \in$ $[-\pi / 2, \pi / 2]$ and $x_{3}(t) \in[-\pi / 2, \pi / 2]$ so that $\Omega$ : $[-\pi / 2, \pi / 2] \times[-\pi / 2, \pi / 2]$. Let the equidistant discretization hyper grid be $M_{1} \times M_{2}: 301 \times 301$ (the transformation space is symmetric on the stabilization point, therefore we set 301 to ensure that one discretization grid line lies on the desired stabilization point). The TP model transformation results in

$\dot{\mathbf{x}}(t)=\left(\sum_{r=1}^{4} w_{r}(\mathbf{p}(t)) \mathbf{A}_{r}\right) \mathbf{x}(t)+\left(\mathbf{B}+\mathbf{D}_{b} \delta_{b} \mathbf{E}_{b}\right) \mathbf{u}(t)$.

The weighting functions $w_{r=1 . .4}(\mathbf{p}(t))$ are depicted on Fig. 7. We can observe that one LTI system dominates in the equilibrium point and the others on the border of $\Omega$. 
The resulting LTI systems are:

$$
\begin{aligned}
\mathbf{A}_{1} & =\left(\begin{array}{ccccc}
0 & 1.0000 & 0 & 0 & 0 \\
47.1020 & 0 & 0 & 0 & 0 \\
0 & 0 & 0 & 1.0000 & 0 \\
0 & 0 & 36.1910 & 0 & 0 \\
0 & 0 & 0 & 0 & 0
\end{array}\right) \\
\mathbf{A}_{2} & =\left(\begin{array}{ccccc}
0 & 1.0000 & 0 & 0 & 0 \\
29.9861 & 0 & 0 & 0 & 0 \\
0 & 0 & 0 & 1.0000 & 0 \\
0 & 0 & 36.1910 & 0 & 0 \\
0 & 0 & 0 & 0 & 0
\end{array}\right) \\
\mathbf{A}_{3} & =\left(\begin{array}{ccccc}
0 & 1.0000 & 0 & 0 & 0 \\
47.1020 & 0 & 0 & 0 & 0 \\
0 & 0 & 0 & 1.0000 & 0 \\
0 & 0 & 23.0399 & 0 & 0 \\
0 & 0 & 0 & 0 & 0
\end{array}\right) \\
\mathbf{A}_{4} & =\left(\begin{array}{ccccc}
0 & 1.0000 & 0 & 0 & 0 \\
29.9861 & 0 & 0 & 0 & 0 \\
0 & 0 & 0 & 1.0000 & 0 \\
0 & 0 & 23.0399 & 0 & 0 \\
0 & 0 & 0 & 0 & 0
\end{array}\right)
\end{aligned}
$$

The conclusion of this subsection is that the simplified uncertain model of the 3-DOF RC helicopter can be exactly given by a convex combination of minimum four LTI systems in such a way that the LTI systems defines the tight convex hull of the given LPV model. This could be derived via analytical works, but the TP model transformation uniformly (independently on the actual analytic form of the given model) results in the polytopic decomposition in a few minutes.

\subsection{Selecting LMI Conditions According to the Control Specifications}

According to the above specifications let us select the following LMI conditions under the PDC framework:

According to (9) we can apply the following simplified LMI conditions for the uncertain TP model (15) to yield robust control:

LMI conditions 1: The simplified uncertain model of the RC helicopter has both $\Delta_{a}=\mathbf{D}_{a}=\mathbf{E}_{a}=0$ and $\mathbf{B}, \Delta_{b}, \mathbf{D}_{b}$ and $\mathbf{E}_{b}$ which do not depend on $\mathbf{p}(t) \in \Omega$. Therefore, we recall the following decay rate condition guaranteeing robust stability condition:

$$
\underset{\mathbf{x}, \mathbf{M}_{1}, d_{b}}{\operatorname{maximize}} \zeta
$$$$
\mathbf{X}, \mathbf{M}_{r}, d_{b}
$$

$$
\text { subject to } \mathbf{X}>\mathbf{0}, \quad d_{b}>0, \quad \hat{\mathbf{S}}_{r r}<\mathbf{0},
$$

where

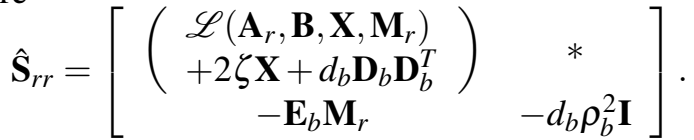

$$
\begin{aligned}
& \mathscr{L}\left(\mathbf{A}_{r}, \mathbf{B}_{r}, \mathbf{X}, \mathbf{M}_{s}\right)=\mathbf{X} \mathbf{A}_{r}^{T}+\mathbf{A}_{r} \mathbf{X}-\mathbf{B}_{r} \mathbf{M}_{s}-\mathbf{M}_{s}^{T} \mathbf{B}_{r}^{T} .
\end{aligned}
$$

for $r<s \leq R$, except the pairs $(r, s)$ such that $\forall \mathbf{p}(t)$ : $w_{r}(\mathbf{p}(t)) w_{s}(\mathbf{p}(t))=0$. The symbol $*$ denotes the transposed elements (matrices) for symmetric positions.

\subsubsection{Constrain on the Control Value}

In order to bound the control value we select the following LMI conditions from [9]:

LMI conditions 2: Assume that initial $\|x(0)\| \leq \phi$, where $x(0)$ is unknown but the upper bound $\phi$ is known. The constraint $\left|u_{j}(t)\right| \leq \mu_{j}(j=1,2, \cdots, m)$ is enforced at all times $t \geq 0$ if there exist a common positive definite matrix $\mathbf{X}$ and matrices $\mathbf{M}_{r}$ such that

$$
\left(\begin{array}{cc}
\phi^{2} I \leq X & \mathbf{M}_{r}^{T} \mathbf{E}_{j}^{T} \\
\mathbf{E}_{j} \mathbf{M}_{r} & \mu_{j}^{2} \mathbf{I}
\end{array}\right) \geq \mathbf{0},
$$

where $\mathbf{E}_{j}$ is the vector whose $j$ th element is one and the other elements are zero, i,e,

$$
\mathbf{E}_{j}=\left[\begin{array}{cccccccc}
1 & j-1 & j & j+1 & & m & \\
0 & \ldots & 0 & 1 & 0 & \ldots & 0
\end{array}\right] .
$$

By using the matrix $\mathbf{E}_{j}$, each input variable can be described as $u_{j}(t)=\mathbf{E}_{j} \mathbf{u}(t)$, where $\mathbf{u}(t)=\left[\begin{array}{llll}u_{1}(t) & u_{2}(t) & \cdots & u_{m}(t)\end{array}\right]^{T}$.

In order to ensure the above condition for a large set of initial states, we can set $\phi$ to be a large quantity even if $x(0)$ is unknown. However, one should note that a large $\phi$ could lead to conservative designs.

The controller with the desired performance is obtained via solving the above LMI conditions 1 and 2 . The solution yields feedback gains $\mathbf{F}_{r}(r=1 \ldots R)$ as:

$$
\mathbf{F}_{r}=\mathbf{M}_{r} \mathbf{X}^{-1} \text {. }
$$

The control value is computed by (18).

In conclusion, the LMI conditions 1 and 2 guarantee the constrain on the control value, good speed of response and avoid input saturations. Since these conditions are represented in terms of LMIs, the feedback gains, satisfying the above multiobjective specification, can be readily obtained numerically, for instance in MATLAB LMI Control Toolbox [16].

\subsection{Numerical Details of the LMI Based Design}

The parameters on input constraint condition are set as follows:

$$
\begin{aligned}
& \mathbf{E}_{1}=\left[\begin{array}{llll}
1 & 0 & 0 & 0
\end{array}\right], \quad \mathbf{E}_{2}=\left[\begin{array}{llll}
0 & 1 & 0 & 0
\end{array}\right], \\
& \mathbf{E}_{3}=\left[\begin{array}{llll}
0 & 0 & 1 & 0
\end{array}\right], \quad \mathbf{E}_{4}=\left[\begin{array}{llll}
0 & 0 & 0 & 1
\end{array}\right], \\
& \mu_{1}=\mu_{3}=0.9 \times 2 \pi f_{0}, \quad \mu_{2}=\mu_{4}=0.1 \times 2 \pi f_{0} .
\end{aligned}
$$

Figure 8 depicts the change of decay rate $\zeta$ for $d_{b}$. The LMI conditions 1 and 2 are feasible in the range from $d_{b}=$ $1.524 \times 10^{7}$ to $3.119 \times 10^{7}$. For the design, we select $d_{b}=$ $2.495 \times 10^{7}$, where the maximum decay rate is obtained:

The resulting LTI feedback systems are:

$\mathbf{F}_{1}=$

$$
\left(\begin{array}{ccccc}
569.3183 & 82.9636 & -32.2101 & -5.3726 & 0.3969 \\
0.3337 & 0.0486 & -0.0188 & -0.0031 & 15.8392 \\
344.5022 & 50.5279 & 694.2790 & 114.5536 & 16.7862 \\
-0.3337 & -0.0486 & 0.0188 & 0.0031 & -15.8392
\end{array}\right)
$$




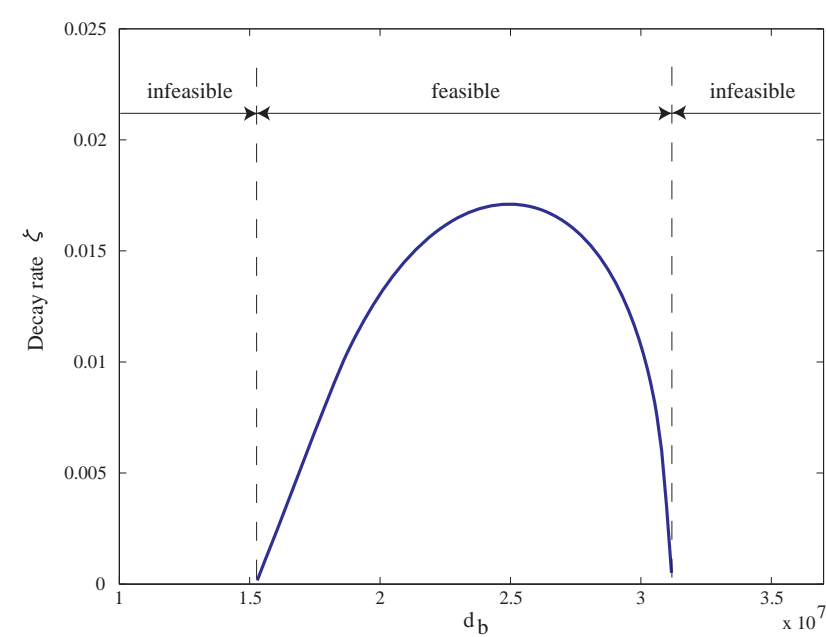

Fig. 8. Decay rate $\zeta$ for $d_{b}$.

$$
\begin{aligned}
& \mathbf{F}_{2}= \\
& \left(\begin{array}{ccccc}
698.6409 & 103.1000 & 97.1139 & 18.0629 & 6.0978 \\
3.4361 & 0.5149 & 1.2268 & 0.2340 & 15.9510 \\
322.4559 & 52.2701 & 588.9798 & 113.3114 & 15.5008 \\
-3.4361 & -0.5149 & -1.2268 & -0.2340 & -15.9510
\end{array}\right) \\
& \mathbf{F}_{3}= \\
& \left(\begin{array}{ccccc}
644.6347 & 102.6238 & 125.1829 & 17.4642 & 12.2166 \\
0.8747 & 0.1391 & 0.3364 & 0.0613 & 12.7815 \\
315.4957 & 49.4383 & 672.3149 & 111.2250 & 14.4206 \\
-0.8747 & -0.1391 & -0.3364 & -0.0613 & -12.7815
\end{array}\right) \\
& \mathbf{F}_{4}= \\
& \left.\begin{array}{ccccc}
526.2796 & 82.5688 & -1.5450 & -5.83206 .8792 \\
10.3008 & 1.6208 & 0.6431 & 0.028013 .0706 \\
256.3282 & 44.8857 & 578.9468 & 111.553312 .6039 \\
-10.3008 & -1.6208 & -0.6431 & -0.0280-13.0706
\end{array}\right)
\end{aligned}
$$

The conclusion of this subsection is that the selected LMI conditions are feasible, the result must guaranty the desired performance. The control value is calculated by (18) as:

$$
\mathbf{u}(t)=-\left(\sum_{r=1}^{4} w_{r}(\mathbf{p}(t)) \mathbf{F}_{r}\right) \mathbf{x}(t) \ldots \ldots .
$$

\section{Control Results}

In this section we present simulation results. We set the initial state values as:

$$
\mathbf{x}(0)=\left[\begin{array}{lllll}
0.279 & 0 & -0.279 & 0 & -0.349
\end{array}\right]^{T} .
$$

Figure 9 shows the stabilization of the 3-DOF RC helicopter (see Fig. 1). One can observe that the decay rate condition guaranteeing the robust stability condition (LMI conditions 1) and the input constraint condition (LMI conditions 2) are satisfied. For comparison, we design a controller guaranteeing only decay rate controller [9] without the robust stability condition. The resulting control performance is presented on Fig. 10. We can observe that the robust controller shows better performance in terms of settling time.

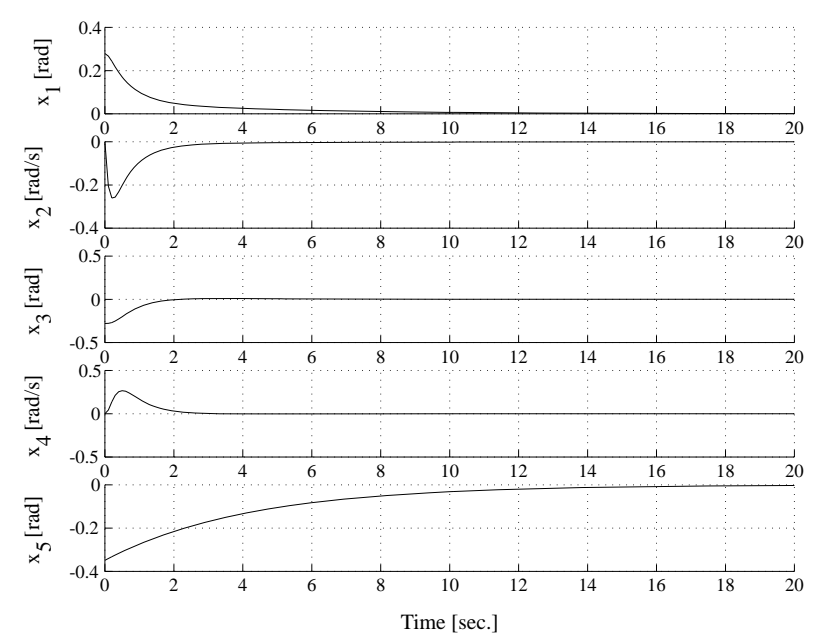

Fig. 9. Control result 1 (robust stability \& decay rate).

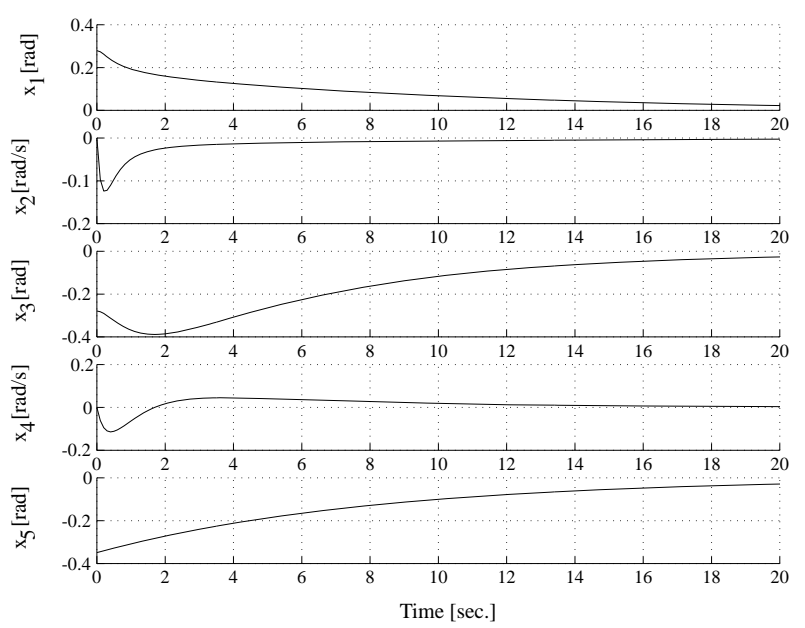

Fig. 10. Control result 2 (decay rate controller).

Remark 2: Since the real operation of the helicopter has influence of ground effect and the lift power loss by deflection of propellers in high frequency rotation which are not involved in the model, the control performance in real experiment may be different. At this point it is worth mentioning that the controllers derived here and in the paper [13] are equivalent. The maximum difference between the outputs of both controllers differ with about $10^{-13}$ for all $\mathbf{p}(t), \mathbf{x}(t)$ (numerically checked). Thus, one should expect the same experimental result applying the presently derived controller as observed in the successful laboratory experiment detailed in paper [13].

\section{Conclusions}

This paper examines the effectiveness of the combination of the TP model transformation and the Parallel Distributed Compensation framework in the stability control design of a real 3-DOF RC helicopter. The paper presents numerical simulations and notices that the derived controller is equivalent with the one successfully tested in laboratory experiment.

The main conclusion of this paper about the design methodology is, that the whole design process is system- 
atic, non-heuristic and straightforward, the stability proof of the resulting controller is tractable via the feasibility test of LMIs and, hence, exact. The whole design procedure is automatically computed via commercialized mathematical tools (MATLAB) without analytical interaction. The computational time is about minutes on a regular PC. It is also worth noticing here, that the same design procedure with the same numerical implementation has been applied to other complex control problems in the related literature as mentioned in the Introduction. This may lead to the conclusion that the proposed design procedure offers a numerical uniform solution for a class of engineering control problems.

\section{Acknowledgements}

This research was supported by the Hungarian National Found OTKA No. F 049838, Dr. Baranyi is supported by János Bolyai Postdoctoral Scholarship. The research was performed in the framework of HUNOROB HU0045 project supported by EEA Grants.

\section{References:}

[1] B. K. Bose, "Neural network applications in power electronics and motor drives-an introduction and perspective," IEEE Transaction on Industrial Electronics, Vol.54, No.1, pp. 14-33, February 2007.

[2] M. Cirrincione, M. Pucci, G. Cirrincione, and G.-A. Capolino, "Sensorless control of induction machines by a new neural algorithm: The TLS EXIN neuron," IEEE Transaction on Industrial Electronics, Vol.54, No.1, pp. 127-149, February 2007.

[3] R. -J. Wai and C. -C. Chu, "Robust petri fuzzy-neural-network control for linear induction motor drive," IEEE Transaction on Industrial Electronics, Vol.54, No.1, pp. 177-189, February 2007.

[4] M. N. Uddin and M. A. Rahman, "High-speed control of ipmsm drives using improved fuzzy logic algorithms," IEEE Transaction on Industrial Electronics, Vol.54, No.1, pp. 190-199, February 2007.

[5] T. Pajchrowski and K. Zawirski, "Application of artificial neural network to robust speed control of servodrive," IEEE Transaction on Industrial Electronics, Vol.54, No.1, pp. 200-207, February 2007.

[6] X. le Wei, J. Wang, and Z. xuan Yang, "Robust smooth-trajectory control of nonlinear servo systems based on neural networks," IEEE Transaction on Industrial Electronics, Vol.54, No.1, pp. 208-217, February 2007.

[7] A. Rubaai, M. Castro-Sitiriche, M. Garuba, and L. Burge, "Implementation of artificial neural network-based tracking controller for high-performance stepper motor drives," IEEE Transaction on Industrial Electronics, Vol.54, No.1, pp. 218-227, February 2007.

[8] P. Baranyi, "TP model transformation as a way to LMI based controller design," IEEE Transaction on Industrial Electronics, Vol.51, No.2, pp. 387-400, April 2004.

[9] K. Tanaka and H. O. Wang, "Fuzzy Control Systems Design and Analysis: A Linear Matrix Inequality Approach," John Wiley \& Sons, Inc., 2001.

[10] Special Session, "TP model transformation in non-linear control," in IEEE International Conference on Fuzzy Systems (FUZZIEEE'04), July 25-29 2004, pp. 1063-1085.

[11] P. Baranyi, "Tensor-product model-based control of twodimensional aeroelastic system," Journal of Guidance, Control, and Dynamics, Vol.29, No.2, pp. 391-400, May-June 2005.

[12] P. Baranyi, P. Zoltán, "Output feedback control of two-dimensional aeroelastic system," Journal of Guidance Control and Dynamics, Vol.29, No.3, pp. 762-767, May-June 2005.

[13] K. Tanaka, H. Ohtake, and H. O. Wang, "A practical design approach to stabilization of a 3-DOF RC helicopter," IEEE Transaction on Control Systems Technology, Vol.12, No.1, pp. 315-325, January 2004.

[14] L. D. Lathauwer, B. D. Moor, and J. Vandewalle, "A multilinear singular value decomposition," SIAM Journal on Matrix Analysis and Applications, Vol.21, No.4, pp. 1253-1278, 2000.

[15] D. Tikk, P. Baranyi, R. J. Patton, and J. Tar, "Approximation properties of TP model forms and its consequences to TPDC design framework," Asian Journal of Control, Vol.9, No.3, pp. 221-231, 2007.
[16] P. Gahinet, A. Nemirovskii, A. J. Laub, and M. Chilali, "LMI Control Toolbox User's Guide," The MathWorks, Inc., 1995.

[17] M.-L. Tomescu, S. Preitl, R.-E. Precup, and J. K. Tar, "Stability Analysis Method for Fuzzy Control Systems Dedicated Controlling Nonlinear Processes," in Acta Polytechnica Hungarica, Vol.4, No.3, pp. 127-141 (ISSN 1785-8860), 2007.

[18] C. Arino and A. Sala, "Relaxed LMI conditions for closed-loop fuzzy systems with tensor-product structure," in Engineering Applications of Artificial Intelligence, Vol.20, pp. 1036-1046, 2007.

[19] F. Kolonic, A. Poljugan, and I. Petrovic, "Tensor Product Model Transformation-based Controller Design for Gantry Crane Control System - An Application Approach," in Acta Polytechnica Hungarica, Vol.3, No.4, pp. 95-112, 2006.

[20] O. Pages and A.E. Hajjaji, "Two fuzzy Multiple Reference Model Tracking Control Designs with an Application to Vehicle Lateral Dynamics Control," European Control Conference on Decision and Control (CDC-ECC '05), pp. 3267-3272, ISBN: 0-7803-9567-0, 12-15 Dec. 2005.

[21] G. Hancke and Á. Szeghegyi: "Nonlinear Control via TP Model Transformation: the TORA System Example," 2nd SlovakianHungarian Joint Symposium on Applied Machine Intelligence (SAMI 2004), Herl'any, Slovakia, pp. 333-340, 16-17 January, 2004.

[22] R.-E. Precup, S. Preitl, J. Fodor, I.-B. Ursache, P.A. Clep, and S Kilyeni, "Experimental validation of iterative feedback tuning solutions for inverted pendulum crane mode control," proc. of Conf. on Human Systems Interaction (HSI 2008), Article no.4581496, pp. 536-541.

[23] R.-E. Precup, S- Preitl, "PI-Fuzzy controllers for integral plants to ensure robust stability," Information Sciences, Vol.177, pp. 44104429, 2007.

[24] R.-E. Precup, Z. Preitl and E.M. Petriu, "Delta domain design of low-cost fuzzy controlled servosystems," proc. of IEEE Int. Symp. on Intelligent Signal Processing (WISP 2007), Article no:4447588. pp. 125-130.

[25] R.-E. Precup, W. S. Lee, M. Venkata, and Zs. Preitl, "Linear and fuzzy control solutions for tape drives," in Electrical Engineering (Archiv für Elektrotechnik), Vol.90, No.5, pp. 361-377, (ISSN 0948-7921), May 2008.

\section{Appendix A.}

The model constants are as follows: $C_{r}=47.1021 / \mathrm{s}^{2}$, $C_{p}=36.1911 / \mathrm{s}^{2}, C_{u r}=5.011 \times 10^{-4}, C_{u p}=5.126 \times$ $10^{-4}, C_{u y}=1.155 \times 10^{-3}, \dot{\theta}_{i \max }=2 \pi f_{0} \mathrm{rad} / \mathrm{s}, \dot{\theta}_{i \text { min }}=$ $0 \mathrm{rad} / \mathrm{s}, \Omega=\pi f_{0} \mathrm{rad} / \mathrm{s}$, where $f_{0}=30 \mathrm{~Hz}$ which is the maximum frequency of the propellers.

\section{Appendix B.}

The equations of motion for the $\mathrm{RC}$ helicopter which is fixed at a joint point are modeled as

$$
\begin{gathered}
M e^{2} \ddot{\gamma}(t)+I_{\gamma} \ddot{\gamma}(t)-M g e \sin \gamma(t) \\
=F_{1}(t) \sqrt{l_{1}^{2}+e^{2}} \cos \left(\tan ^{-1} \frac{e}{l_{1}}\right) \\
\quad-F_{3}(t) \sqrt{l_{1}^{2}+e^{2}} \cos \left(\tan ^{-1} \frac{e}{l_{1}}\right) \ldots . . \\
M e^{2} \ddot{\beta}(t)+I_{\beta} \ddot{\beta}(t)-M g e \sin \beta(t) \\
=-F_{2}(t) \sqrt{l_{2}^{2}+e^{2}} \cos \left(\tan ^{-1} \frac{e}{l_{2}}\right) \\
\quad+F_{4}(t) \sqrt{l_{2}^{2}+e^{2}} \cos \left(\tan ^{-1} \frac{e}{l_{2}}\right) \ldots . . \\
\left(I_{\alpha}+4 I_{1}\right) \ddot{\alpha}(t)=I_{1}\left(\ddot{\theta}_{1}(t)-\ddot{\theta}_{2}(t)+\ddot{\theta}_{3}(t)-\ddot{\theta}_{4}(t)\right)
\end{gathered}
$$


where $\gamma(t), \beta(t)$ and $\alpha(t)$ are the angles of roll, pitch and yaw, respectively, $M$ is the mass of the helicopter. $I_{\gamma}, I_{\beta}$ and $I_{\alpha}$ are the moments of inertia around $x, y$ and $z$-axes with respect to the gravity point of the helicopter, respectively. $I_{1}$ is the moment of inertia of a propeller. $g$ is the gravity constant and $e, l_{1}$ and $l_{2}$ are lengths shown in Fig. 3-6. $F_{i}$ is the lift force generated by the $i$ th propeller. It is described as

$$
F_{i}(t)=\frac{1}{3} C_{L} \rho S l_{w}^{2} \dot{\theta}_{i}^{2}(t) \text {. . . . . . . . . . }
$$

where $C_{L}$ is the lift coefficient, $\rho$ is the air density, $S$ is the area of a wing of each propeller, $l_{w}$ is the length of a wing and $\dot{\theta}_{i}(t)$ is the $i$ th propeller's angular velocity. We assume from the property of the motors (of the propellers) that $\dot{\theta}_{i}(t) \geq 0$ for all $i$.

From (23), (24), (25) and (26),

$$
\begin{aligned}
& \ddot{\gamma}(t)=C_{r} \sin \gamma(t)+C_{u r}\left(\dot{\theta}_{1}^{2}(t)-\dot{\theta}_{3}^{2}(t)\right) . . \\
& \ddot{\beta}(t)=C_{p} \sin \beta(t)+C_{u p}\left(-\dot{\theta}_{2}^{2}(t)+\dot{\theta}_{4}^{2}(t)\right) . \\
& \ddot{\alpha}(t)=C_{u y}\left(\ddot{\theta}_{1}(t)-\ddot{\theta}_{2}(t)+\ddot{\theta}_{3}(t)-\ddot{\theta}_{4}(t)\right) .
\end{aligned}
$$

where $C_{r}, C_{p}, C_{u r}, C_{u p}$ and $C_{u y}$ are constant values which consist of $M, I_{\gamma}, I_{\beta}, I_{\alpha}, I_{1}, g, e, l_{1}, l_{2}, C_{L}, \rho$ and $S$. By integrating (29) with respect to time $t$, we obtain

$$
\begin{aligned}
& \dot{\alpha}(t)-\dot{\alpha}(0)=C_{u y}\left\{\left(\dot{\theta}_{1}(t)-\dot{\theta}_{1}(0)\right)-\left(\dot{\theta}_{2}(t)-\dot{\theta}_{2}(0)\right)\right. \\
& \left.+\left(\dot{\theta}_{3}(t)-\dot{\theta}_{3}(0)\right)-\left(\dot{\theta}_{4}(t)-\dot{\theta}_{4}(0)\right)\right\} \quad . \quad . \quad . \quad .
\end{aligned}
$$

where $\dot{\alpha}(0), \dot{\theta}_{1}(0), \dot{\theta}_{2}(0), \dot{\theta}_{3}(0)$ and $\dot{\theta}_{4}(0)$ are initial angular velocities. Assume that $\dot{\alpha}(0)=0$. If $\dot{\theta}_{1}(0)=\dot{\theta}_{2}(0)=$ $\dot{\theta}_{3}(0)=\dot{\theta}_{4}(0),(30)$ can be rewritten as follows:

$$
\dot{\alpha}(t)=C_{u y}\left(\dot{\theta}_{1}(t)-\dot{\theta}_{2}(t)+\dot{\theta}_{3}(t)-\dot{\theta}_{4}(t)\right) \ldots
$$

Assume that $\Omega$ is an equilibrium point of the propeller's angular velocity. The relation between $\Omega$ and $\dot{\theta}_{i}(t)$ is given as

$$
\dot{\theta}_{i}(t)=\Omega+\Delta \dot{\theta}_{i}(t)
$$

where $\Delta \dot{\theta}_{i}(t)$ is the change of the $i$ th propeller's angular velocity around $\Omega$. From (32), $\Delta \dot{\theta}_{i}(t) \in\left[\dot{\theta}_{i \min }-\right.$ $\left.\Omega \quad \dot{\theta}_{i \max }-\Omega\right]$, where $\dot{\theta}_{i \max }$ and $\dot{\theta}_{i \text { min }}$ denote the maximum and minimum values of $\dot{\theta}_{i}(t)$, respectively, where $\dot{\theta}_{i \max }>\dot{\theta}_{i \min } \geq 0$. We note that any $\Omega$ satisfying $\max _{i} \dot{\theta}_{i \min } \leq \Omega \leq \min _{i} \dot{\theta}_{i \max }$ can be selected due to the structure of this type of helicopters with four propellers. Using (32), the equations of motion (27), (28) and (31) can be rewritten as follows.

$$
\begin{aligned}
\ddot{\gamma}(t)= & C_{r} \sin \gamma(t)+C_{u r}\left(2 \Omega+\Delta \dot{\theta}_{1}(t)+\Delta \dot{\theta}_{3}(t)\right) \\
& \times\left(\Delta \dot{\theta}_{1}(t)-\Delta \dot{\theta}_{3}(t)\right) \cdot . . . . \\
\ddot{\beta}(t)= & C_{p} \sin \beta(t)+C_{u p}\left(2 \Omega+\Delta \dot{\theta}_{2}(t)+\Delta \dot{\theta}_{4}(t)\right) \\
& \times\left(-\Delta \dot{\theta}_{2}(t)+\Delta \dot{\theta}_{4}(t)\right) . . . . . \\
\dot{\alpha}(t)= & C_{u y}\left(\Delta \dot{\theta}_{1}(t)+\Delta \dot{\theta}_{3}(t)-\Delta \dot{\theta}_{2}(t)-\Delta \dot{\theta}_{4}(t)\right) .
\end{aligned}
$$

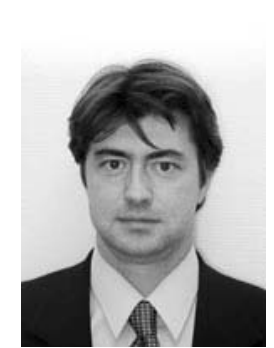

Name:

Péter Baranyi

Affiliation:

Computer and Automation Research Institute, Hungarian Academy of Sciences

Address:

H-1111 Budapest, Kende utca 13-17, Hungary

Brief Biographical History:

1994 M.Sc. degree in electrical engineering, Budapest University of Technology and Economics

1995 M.Sc. degree in education of engineering sciences, Budapest University of Technology and Economics

1999 Ph.D. degree, Budapest University of Technology and Economics 2006 D.Sc., degree, Doctor of the Hungarian Academy of Sciences

Membership in Academic Societies:

- Founding member of the Integrated Intelligent Systems

Japanese-Hungarian Laboratory

- Founding member of the ITM Norwegian-Hungarian Laboratory

- Member of IEEE

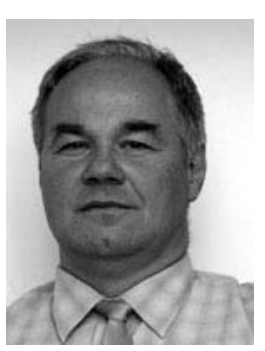

\section{Name:}

Péter Korondi

\section{Affiliation:}

Department of Automation and Applied Informatics, Budapest University of Technology and Economics (BUTE)

\section{Address:}

Goldmann György tér 3. H-1111 Budapest, Hungary

Brief Biographical History:

1984-1986 Ganz Meter Factory: R\&D engineer

1986-1993 BUTE: Assistant

1993-1995 University of Tokyo: Postgraduate student

1995-1997 BUTE: Senior lecturer

1997- BUTE: Associate professor

\section{Main Works:}

- P. Korondi, "Sliding Mode Based Friction Compensation for a Haptic Interface," Strojarstvo., 48, 3-4, pp. 163-171, 2006.

- N. Ando, P. Korondi, and H. Hashimoto "Networked

Tele-micromanipulation Systems "Haptic Loupe," IEEE

TRANSACTIONS ON INDUSTRIAL ELECTRONICS, Vol.51(6), pp. 1259-1271, December, 2004.

- P. Korondi, H. Hashimoto, and V. Utkin, "Direct Torsion Control of Flexible Shaft based on an Observer Based Discrete-time Sliding Mode," IEEE Trans. on Industrial Electronics, Vol.IE, No.2, pp. 291-296, 1998.

Membership in Academic Societies:

- IEEE Industrial Electronics Society

- IEEE Industry Application Society 
Baranyi, P., Korondi, P., and Tanaka, K.

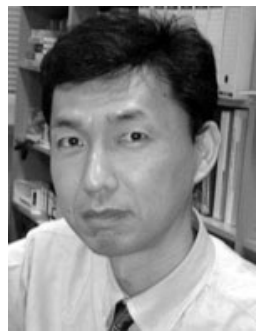

Name:

Kazuo Tanaka

\section{Affiliation:}

Professor, Dept. of Mechanical Engineering and Intelligent Systems, The University of ElectroCommunications

Address:

1-5-1 Chofugaok, Chofu, Tokyo 182-8585, Japan

Brief Biographical History:

1990- Ph.D., Systems Science, Tokyo Institute of Technology

Main Works:

- "Fuzzy Control Systems Design and Analysis: A Linear Matrix

Inequaility," Wiley-Intersciences, 2001.

Membership in Academic Societies:

- Institute of Electrical and Electronics Engineers (IEEE)

- The Society of Instrument and Control Engineers (SICE)

- The Robotics Society of Japan (RSJ) 